Archive for

Organic Chemistry

Arkivoc 2019, part vi, 200-211

\title{
Samarium triflate-catalyzed dimerization of vinylarenes
}

\author{
Meng-Yang Chang*a,b and Yu-Lin Tsai ${ }^{a}$ \\ ${ }^{a}$ Department of Medicinal and Applied Chemistry, Kaohsiung Medical University, Kaohsiung 807, Taiwan \\ ${ }^{b}$ Department of Medical Research, Kaohsiung Medical University Hospital, Kaohsiung 807, Taiwan
}

Email: mychang@kmu.edu.tw

Received 09-28-2019

Accepted 11-05-2019

Published on line 11-30-2019

\section{Abstract}

We report the preparation of substituted indanes and their dimeric isomers via the samarium triflatemediated [Sm(OTf) 3,10 mol\%] self-dimerization of vinylarenes in $\mathrm{MeNO}_{2}$ at $25{ }^{\circ} \mathrm{C}$ for $10 \mathrm{~h}$. The diverse products were obtained in moderate to high yields. The synthesis involves a $(3+2)$ annulation via the formation of carbon-carbon bonds. Plausible mechanisms are proposed and discussed. The investigation of various rare metal triflates catalyst loadings, reaction conditions, and substrate scope led to an operationally easy one-pot Friedel-Crafts reaction protocol.

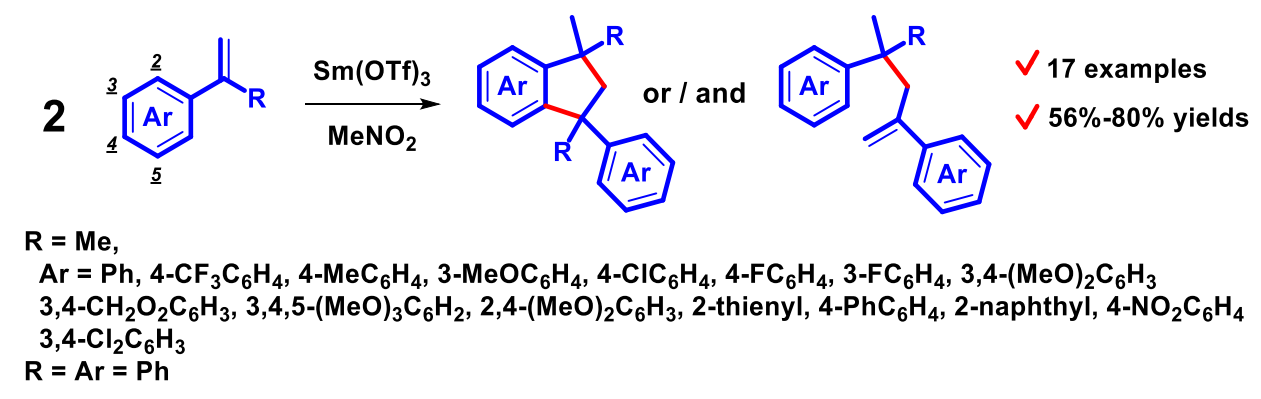

Keywords: Samarium triflate, vinylarenes, indane, dimerization 


\section{Introduction}

The catalytic self-dimerization reaction of vinyl arenes (e.g. $\alpha$-methylstyrenes) to derive functionalized indanes is one of the most straightforward and useful transformations used to construct carbon-carbon (C-C) bonds. After pioneer work by Bergmann and co-workers, ${ }^{1}$ various promoter-mediated synthetic routes have been documented via the intermolecular hydroarylation of vinyl arenes followed by spontaneous intramolecular ring-closure of the resulting dimeric isomers (Scheme 1$)$. They include different metal-free reagents $\left(I_{2}, T F A\right.$, aminium salt) $)^{2-5}$ transition-metal complexes $\left(\mathrm{In}^{3+}, \mathrm{Bi}^{3+}, \mathrm{Ru}^{3+}-\mathrm{Ru}^{+4}, \mathrm{Pd}^{2+} / \mathrm{In}^{3+}, \mathrm{Eu}^{3+}, \mathrm{Au}^{3+}, \mathrm{Mo}^{2+}, \mathrm{Ce}^{3+}\right),{ }^{6-13}$ nanoparticles (MCM-41, Al- $-\mathrm{SiO}_{2}, \mathrm{H}_{3} \mathrm{PW}_{12} \mathrm{O}_{40} / \mathrm{SiO}_{2}$, Nafion) $)^{14-17}$ or other methods. ${ }^{18-21}$ On the basis of observations, attempts to develop new and efficient catalyst systems for the self-dimerization of vinyl arenes are still in demand. In an ongoing effort to emphasize the synthetic applications of metal triflates, ${ }^{22-28}$ we present, herein, a $\operatorname{Ln}(\mathrm{OTf})_{3}$ (lanthanide triflate)-mediated synthesis of substituted indanes. ${ }^{29-30}$ To the best of our knowledge, no examples have been reported for $\operatorname{Ln}(\mathrm{OTf})_{3}$-mediated self-dimerizations of this type.

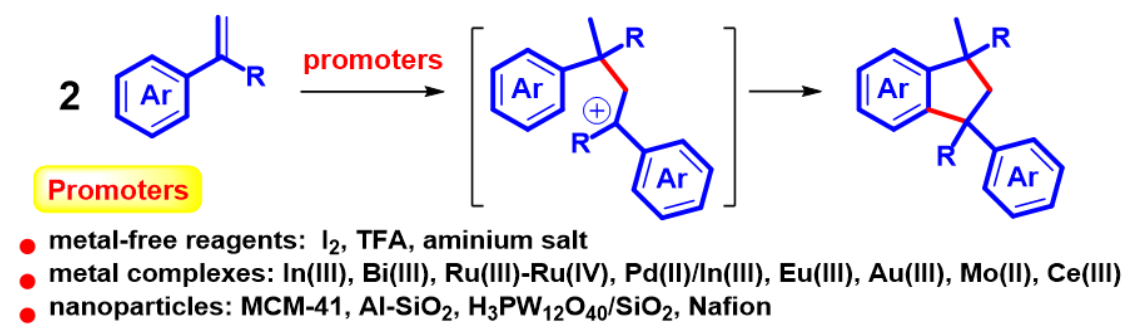

Scheme 1. Self-dimerization of vinyl arenes.

\section{Results and Discussion}

After perusing literature on the synthesis of substituted indanes and reviewing our previous studies on metal triflate (1)-promoted reactions, 10 commercially available $\operatorname{Ln}(\mathrm{OTf})_{3} \mathbf{2 a}-\mathbf{2 j} \mathbf{j}(10 \mathrm{~mol} \%)$ promoted dimerizations of starting $\alpha$-methylstyrene (3a) were examined in $\mathrm{MeNO}_{2}(5 \mathrm{~mL})$ at $25{ }^{\circ} \mathrm{C}$ for $5 \mathrm{~h}$. However, no obvious yield changes occurred with the isolation of $4 \mathbf{a}$ using $5 \mathrm{~mol} \%$ of $\mathrm{La}(\mathrm{OTf})_{3}(\mathbf{2 a}), \mathrm{Ce}(\mathrm{OTf})_{3}(\mathbf{2 b}), \operatorname{Pr}(\mathrm{OTf})_{3}(\mathbf{2 c}), \mathrm{Nd}(\mathrm{OTf})_{3}$ (2d), Sm(OTf) $)_{3}(\mathbf{2 e}), \mathrm{Eu}(\mathrm{OTf})_{3}(\mathbf{2 f}), \mathrm{Gd}(\mathrm{OTf})_{3}(\mathbf{2 g}), \mathrm{Dy}(\mathrm{OTf})_{3}(\mathbf{2 h}), \mathrm{Er}(\mathrm{OTf})_{3}(\mathbf{2 i})$ or Yb(OTf) $)_{3}(\mathbf{2 j})$. Only Sm(OTf) $)_{3}(\mathbf{2 e})$ and $\mathrm{Gd}(\mathrm{OTf})_{3}(\mathbf{2 g})$ provided $4 \mathrm{a}$ in better $(66 \%$ and $47 \%)$ yields, respectively. Other catalysts produced $4 \mathrm{a}$ in low yields (trace amounts to $20 \%$ ). On the basis of the results, $\mathrm{Sm}(\mathrm{OTf})_{3}(\mathbf{2 e})$ was controlled as a catalyst to screen the optimal conditions (Table 1, entry 1). For comparing the advantages of the shown synthetic procedure with others previously described in the literature (e.g. $\ln B r_{3},{ }^{6} \mathrm{BiCl}_{3}{ }^{7}$ or $\mathrm{TFA}^{18}$ ), we found that $\mathrm{Sm}(\mathrm{OTf})_{3}$ was the first lanthanide catalyst to promote the formation of substituted indanes via self-dimerization of $\alpha$ methylstyrene. Nevertheless, $\mathrm{Sm}(\mathrm{OTf})_{3}$ is relatively expensive because samarium is a rare metal resource.

Further variations in the reaction parameters, such as catalyst loading, the solvent system, temperature and reaction time, were carried out as follows. In entry 2, decreasing the catalytic equivalent of $\mathrm{Sm}(\mathrm{OTf})_{3}(10 \rightarrow 5$ mol\%) diminished the yield of $4 a$ ( $66 \rightarrow 39 \%$ ). Entry 3 showed that excess amounts ( 20 mol\%) of Sm(OTf) $)_{3}$ did not increase the catalytic ability to provide a better yield, and a similar yield (64\%) was observed. After elevating the temperature $\left(25 \rightarrow 75^{\circ} \mathrm{C}\right)$, 4a was isolated in only a $41 \%$ yield (entry 4$)$. Under a refluxing $\mathrm{MeNO}_{2}\left(101{ }^{\circ} \mathrm{C}\right)$ condition, $4 \mathrm{a}$ was isolated in a low yield $(35 \%)$, and a very complex mixture was detected, as shown in entry 5 . With longer reaction times $(5 \rightarrow 10,20 \mathrm{~h}$ ), 4a was formed in higher yield (83\%, 80\%) (entries 
6-7). Furthermore, controlling the reaction conditions for the combination of $\mathrm{Sm}(\mathrm{OTf})_{3}(10 \mathrm{~mol} \%), 25{ }^{\circ} \mathrm{C}$ and

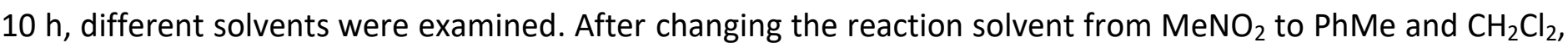
$4 a$ was produced in lower yields $(28 \%, 60 \%)$ than $\mathrm{MeNO}_{2}$ (entries 8 \& 9). In particular, entry 10 showed that the use of DMF did not give the desired product 4a. On the basis of TLC monitoring, only the starting material $3 a$ was detected. However, treatment of $3 a$ with diluted $\mathrm{MeNO}_{2}$ afforded $4 \mathrm{a}$ in a similar yield (80\%) (entry 11 ). This meant that the reaction concentration was not a main factor affecting the 4a yield.

Table 1. Optimal conditions ${ }^{a-b}$

\begin{tabular}{|c|c|c|c|c|c|}
\hline entry & $\mathrm{Sm}(\mathrm{OTf})_{3}(\mathrm{~mol} \%)$ & solvent $(\mathrm{mL})$ & temp $\left({ }^{\circ} \mathrm{C}\right)$ & time $(\mathrm{h})$ & yield $^{b}(\%)$ \\
\hline 1 & 10 & $\mathrm{MeNO}_{2}$ & 25 & 5 & 66 \\
\hline 2 & 5 & $\mathrm{MeNO}_{2}$ & 25 & 5 & 39 \\
\hline 3 & 20 & $\mathrm{MeNO}_{2}$ & 25 & 5 & 64 \\
\hline 4 & 10 & $\mathrm{MeNO}_{2}$ & 75 & 5 & 41 \\
\hline 5 & 10 & $\mathrm{MeNO}_{2}$ & 101 & 5 & 35 \\
\hline 6 & 10 & $\mathrm{MeNO}_{2}$ & 25 & 10 & 83 \\
\hline 7 & 10 & $\mathrm{MeNO}_{2}$ & 25 & 20 & 80 \\
\hline 8 & 10 & PhMe & 25 & 10 & $28^{c}$ \\
\hline 9 & 10 & $\mathrm{CH}_{2} \mathrm{Cl}_{2}$ & 25 & 10 & 60 \\
\hline 10 & 10 & DMF & 25 & 10 & $\mathrm{ND}^{d}$ \\
\hline 11 & 10 & $\mathrm{MeNO}_{2}{ }^{e}$ & 25 & 10 & 80 \\
\hline
\end{tabular}

${ }^{a}$ Reaction was run on 3 a $(1.0 \mathrm{mmol})$, solvent $(5 \mathrm{~mL}) .{ }^{b}$ Isolated yields. ${ }^{c}$ Complex reaction mixture (TLC). ${ }^{d}$ No reaction. ${ }^{e}$ Solvent $(10 \mathrm{~mL})$.

On the basis of the highest observed yield (entry 6, 83\%), the combination of $10 \mathrm{~mol} \% \mathrm{Sm}(\mathrm{OTf})_{3} / \mathrm{MeNO}_{2}(5$ $\mathrm{mL} / 25{ }^{\circ} \mathrm{C} / 10 \mathrm{~h}$ was selected as the optimal reaction conditions for the formation of dimer $4 \mathrm{a}$. On the other hand, $\mathrm{Sm}(\mathrm{OTf})_{3}$ has been reported as a catalyst for different reaction types, including Ferrier rearrangement, ${ }^{31}$ Friedel-Crafts alkylation, ${ }^{32}$ aza-Diels-Alder cycloaddition, ${ }^{33}$ conjugated addition ${ }^{34}$ and cross-coupling. ${ }^{35}$ Remarkably, there are few examples of samarium salt-catalyzed reactions having been performed in comparison with other commercially available samarium complexes. ${ }^{36-40}$ With the optimal reaction conditions in hand (Table 1, Entry 6), we then explored the scope of the conversion with other substrates (Table 2).

For the aryl substituent of $\alpha$-methylvinyl arenes 3a-p, the Ar ring with diversified electron-neutral, electrondonating or electron-withdrawing groups was examined next, including a Ph, b 4- $\mathrm{CF}_{3} \mathrm{C}_{6} \mathrm{H}_{4}, \mathbf{c} 4-\mathrm{MeC}_{6} \mathrm{H}_{4}, \mathbf{d} 3-$ $\mathrm{MeOC}_{6} \mathrm{H}_{4}$, e 4- $\mathrm{ClC}_{6} \mathrm{H}_{4}$, f 4- $\mathrm{FC}_{6} \mathrm{H}_{4}$, g 3- $\mathrm{FC}_{6} \mathrm{H}_{4}$, h 3,4- $(\mathrm{MeO})_{2} \mathrm{C}_{6} \mathrm{H}_{3}$, i 3,4- $\mathrm{CH}_{2} \mathrm{O}_{2} \mathrm{C}_{6} \mathrm{H}_{3}$, j 3,4,5-(MeO) ${ }_{3} \mathrm{C}_{6} \mathrm{H}_{2}$, $k$ 2,4-

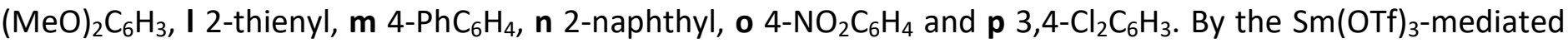
self-dimerization of 3a-p, two kinds of dimers, cyclized indanes (4a, $\mathbf{4 c - n}$ ) and acyclic isomers (4b-1, 4e-1, 4f-1, 4g-1, 4o-1, 4p-1), were provided in a range of $56-83 \%$ and $8 \%-65 \%$ yields, respectively. In all examples, the four electron-neutral aryl groups (for 3a, 3c, 3m-n), five electron-donating oxygenated aryl groups (for $\mathbf{3 d}$, 3hk) and one heterocyclic group (for $\mathbf{3} \mathbf{l}$ ) could trigger the ring-closure to produce a benzo-fused indane skeleton. 
However, six electron-withdrawing aryl groups (for $\mathbf{3 b}, \mathbf{3 e - f}, \mathbf{3 g}, \mathbf{3 0}-\mathbf{p}$ ) afforded an acyclic dimer or a mixture of a cyclic indane and acyclic dimer. One reason for this is the electron-deficient aryl group does not have enough electron density to promote spontaneous annulation such that sole acyclic dimers would form, especially for 4-trifluoromethylphenyl, 4-nitrophenyl and 3,4-dichlorophenyl groups. However, another three weaker electron-withdrawing aryl groups (fluoro-, chloro-) obtained a mixture of a cyclic indane and acyclic dimer. Among these products $\mathbf{4}$, only $\mathbf{3 d}\left(\mathrm{Ar}=3-\mathrm{MeOC}_{6} \mathrm{H}_{4}\right)$ provided the unseparated mixture of products $\mathbf{4 d}$ (for 3$\mathrm{MeO}$ ) and 4d' (for 5-MeO) with a ratio of 80:20. During the ring-closure procedure, the methoxy group at the

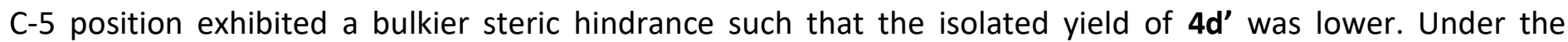

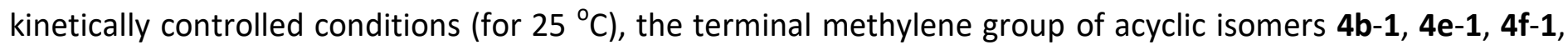
4g-1, 4o-1 and 4p-1 could demonstrate that exo-chemoselectivity. The similar phenomenon had described by Peppe and Nishibayashi groups, respectively. ${ }^{6,8}$

Table 2. $\mathrm{Sm}(\mathrm{OTf})_{3}$-catalyzed self-dimerization of $3^{a-b}$

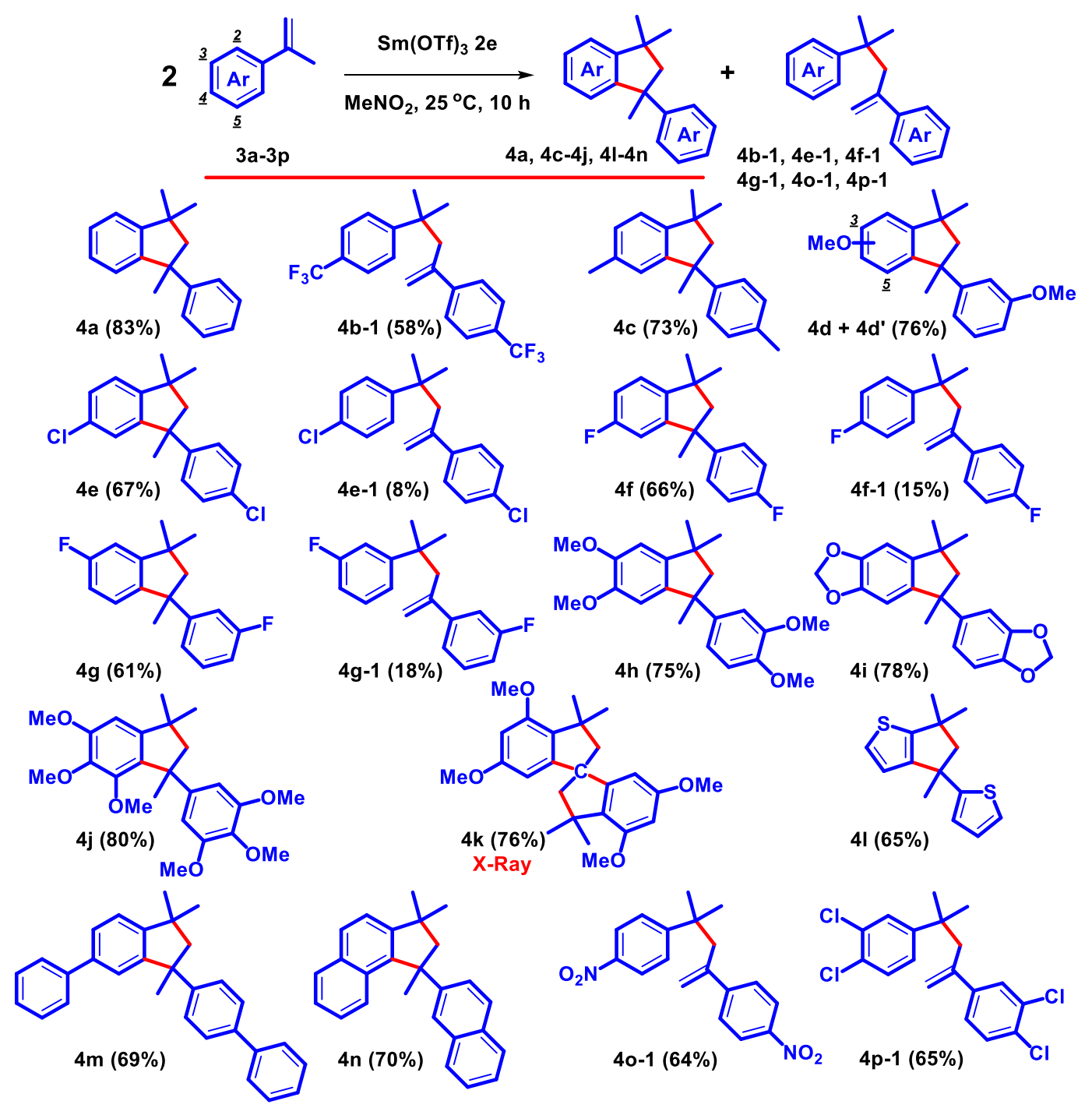

${ }^{a}$ The synthesis was run on $1.0 \mathrm{mmol}$ scale with $3 \mathrm{a}-\mathrm{p}, \mathrm{Sm}(\mathrm{OTf})_{3}(60 \mathrm{mg}, 10 \mathrm{~mol} \%)$, $\mathrm{MeNO}_{2}(5 \mathrm{~mL}), 10 \mathrm{~h}, 25{ }^{\circ} \mathrm{C} .{ }^{b}$ Isolated yield. 
$\alpha$-Phenylstyrene (3q) was also studied (Table 2). On the basis of the styrene skeleton, changing the $\alpha$-methyl group to $\alpha$-phenyl was tested. Under the above-mentioned conditions, treatment of $\mathbf{3 q}$ with $\mathrm{Sm}(\mathrm{OTf})_{3}$ afforded $4 q$ in a $56 \%$ yield along with a $25 \%$ yield of unknown and unanalyzed products mixture (Scheme 2 ).

2<smiles>C=C(c1ccccc1)c1ccccc1</smiles>

$3 q$

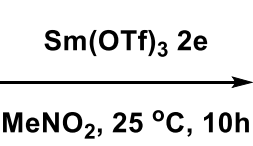

$\mathrm{MeNO}_{2}, 25^{\circ} \mathrm{C}, 10 \mathrm{~h}$

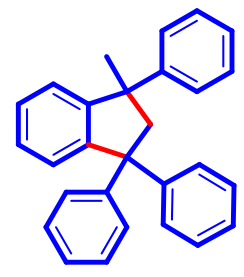

$4 q(56 \%)$

Scheme 2. Synthesis of indane $\mathbf{4 q}$.

Based on experimental results, a possible reaction mechanism with both electron-withdrawing 4trifluoromethylphenyl group and electron-donating 3,4-dimethoxyphenyl groups is shown in Scheme 3 . How were two dimers $\mathbf{4 b - 1}$ and $\mathbf{4 h}$ produced? The event is initiated to form $\mathbf{A}$ by complexation of an olefinic moiety of 3 with $\mathrm{Sm}(\mathrm{OTf})_{3}$. After releasing a triflate anion, B, with a methylene samarium arm, is generated. Then, participation of another $\mathbf{3}$ converts the resulting $\mathbf{B}$ into $\mathbf{C}$ having a tertiary carbocation. On the basis of the structure on $\mathbf{C}$, path a (green) shows that the $4-\mathrm{CF}_{3}$ group decreases the electron density of Ar such that the triflate anion could trap the proton to stabilize carbocation. Following in-situ formed triflic acid-promoted protodemetalation of $\mathbf{D}$, the removal of $\mathrm{Sm}(\mathrm{OTf})_{3}$ afforded $\mathbf{4 b - 1}$. For path $b$ (red), owing to $\mathrm{Ar}=3,4-(\mathrm{MeO})_{2}$, the electron-rich arene could attack the carbocation to give $\mathbf{E}$ via the five-membered ring closure procedure. Following the triflate anion-mediated dehydrogenative aromatization of $\mathbf{E}$, and then, triflic acid-promoted protodemetalation of $\mathbf{F}$, tetramethoxyindane $\mathbf{4 h}$ is obtained along with the recovery of $\mathrm{Sm}(\mathrm{OTf})_{3}$. From the plausible mechanisms, we understand that electron-density on arene is a key factor in affecting the reaction pathway and product distribution.

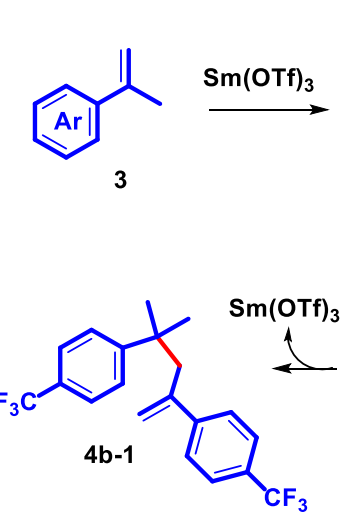

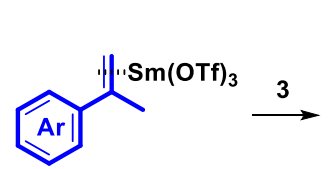

A
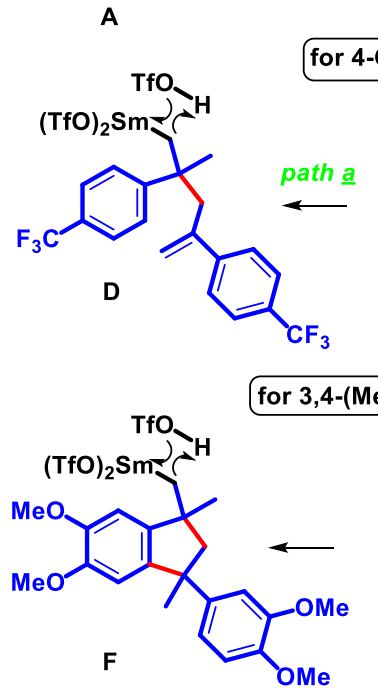
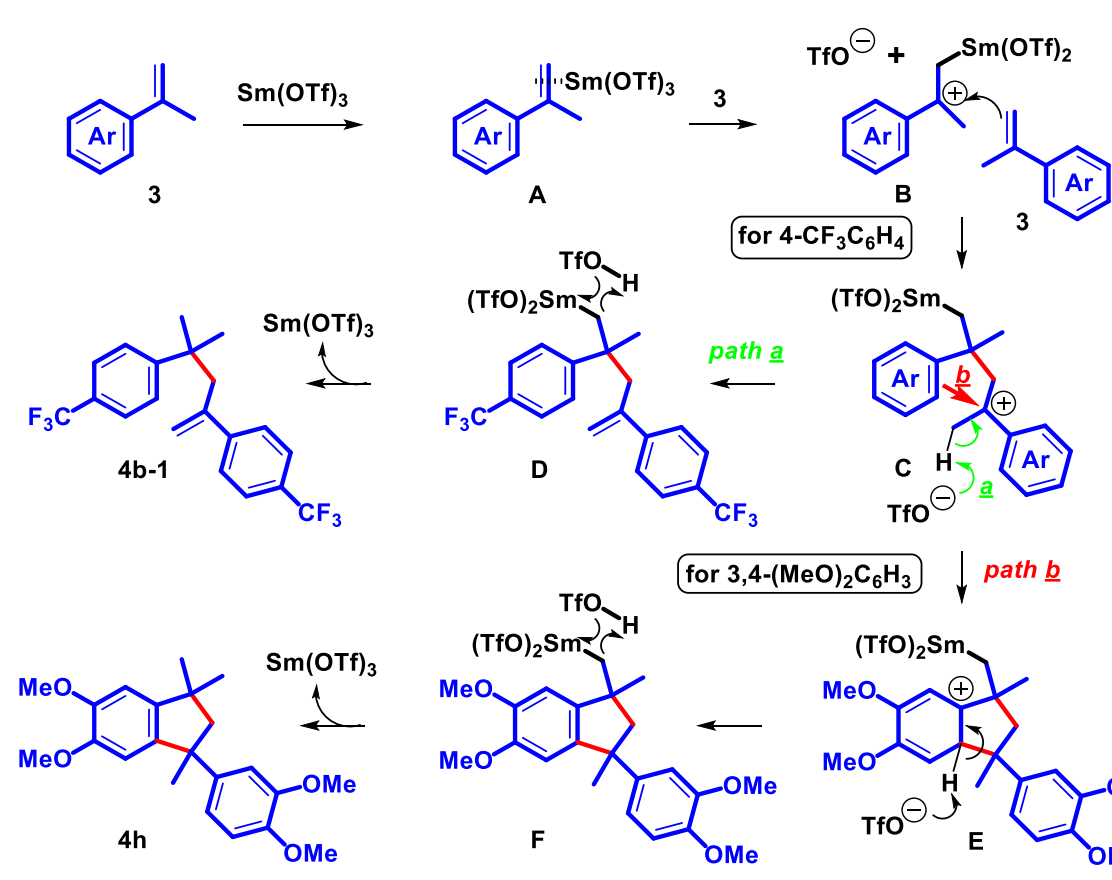

Scheme 3. Plausible mechanism. 
In particular, when Ar with a 2,4-dimethoxy group was treated with the above reaction conditions, only $4 \mathbf{k}$ was generated in a $76 \%$ yield. The spiro structure of bis-indane $\mathbf{4 k}$ was determined by single-crystal $\mathrm{X}$-ray crystallography. ${ }^{41}$ We postulated that three equivalents of $\mathbf{3 k}$ were involved to generate $\mathbf{4 k}$ (Scheme 4). According to a series of reaction steps in Scheme 3, path $b$, II is produced from I with an oxygen-chelated samarium complex conformation. By removal of 1,3-dimethoxybenzene, III should be formed. This is a very important step for the formation of a bis-indane skeleton because the formed 3-carbon fragment could construct a spiro ring. Furthermore, intermolecular Friedel-Crafts type coupling of the corresponding III with another I produces IV. Next, electron-rich arene attacks the carbocation to give $\mathbf{V}$ via intramolecular benzannulation. Following the above-mentioned steps (triflate anion-mediated dehydrogenative aromatization of $\mathbf{V}$ followed by triflic acid-promoted protodemetalation of $\mathbf{V I}$ ), tetramethoxy spiro-indane $\mathbf{4} \mathbf{k}$ is obtained along with the recovery of $\mathrm{Sm}(\mathrm{OTf})_{3}$. Compared with the formation of oxygenated indanes $\mathbf{4 h}-\mathbf{k}$, only $\mathbf{4 k}$ was produced as a spiro system under similar reaction conditions. The detailed reasons are still unclear, however, we believe that the 2-MeO group of $\mathbf{3 k}$ plays a role in triggering the removal of 1,3dimethoxybenzene more easily than other oxygenated vinylarenes $\mathbf{3 h}$-j during the conversion process from II to III.

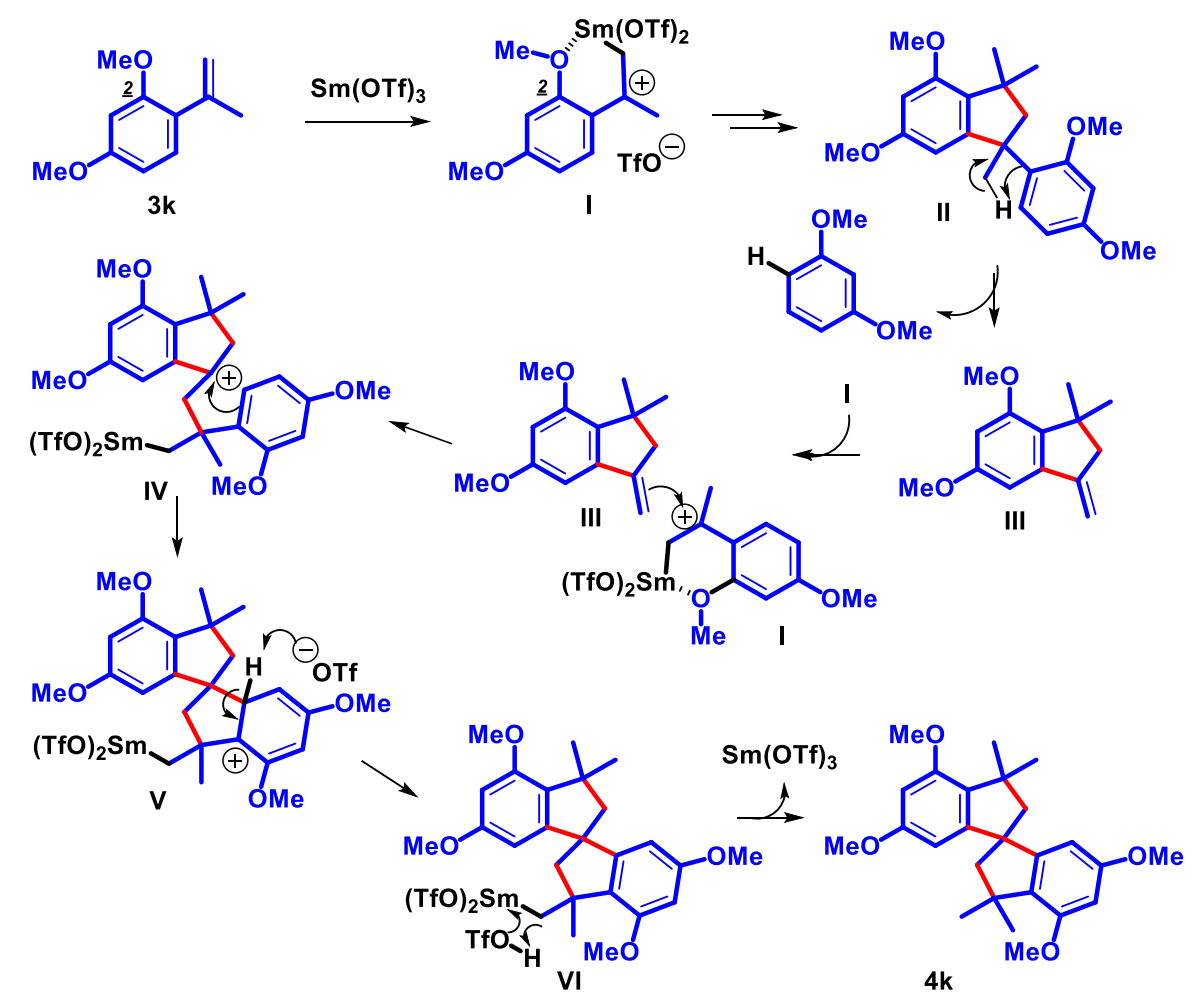

Scheme 4. Plausible Mechanism of $\mathbf{4 k}$.

\section{Conclusions}

We have developed a mild synthesis of substituted indanes and dimeric isomers in moderate yields via a 10

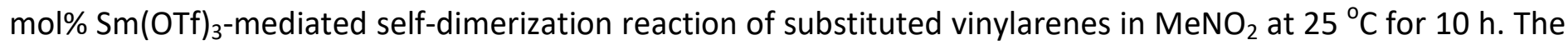
control of reaction parameters such as the lanthanide triflates catalyst loading, the reaction temperature, the solvent and the time, had to be finely tuned to explore optimal conditions. Furthermore, the proposed 
mechanisms for the formation of $\mathbf{4 b - 1}, \mathbf{4 h}$ and $\mathbf{4 k}$ are discussed. Further investigation regarding synthetic applications of lanthanide triflates will be conducted and published in due course.

\section{Experimental Section}

General. All reagents and solvents were obtained from commercial sources and used without further purification. Reactions were routinely carried out under an atmosphere of air with magnetic stirring. Products in organic solvents were dried with anhydrous magnesium sulfate before concentration in vacuo. All reactions were monitored by TLC on silica gel $60 \mathrm{~F}_{254}$ (Merck) with detection by UV light. Column chromatography was performed using silica gel (200-300 mesh). Melting points were determined with a SMP3 melting apparatus. ${ }^{1} \mathrm{H}$ and ${ }^{13} \mathrm{C}$ NMR spectra were recorded on a Varian INOVA-400 spectrometer operating at 400 and $100 \mathrm{MHz}$, respectively. Chemical shifts $(\delta)$ are reported in parts per million (ppm) and the coupling constants $(J)$ are given in Hertz $(\mathrm{Hz})$. High resolution mass spectra (HRMS) were measured with a mass spectrometer Finnigan/Thermo Quest MAT 95XL. X-ray crystal structures were obtained with an Enraf-Nonius FR-590 diffractometer (CAD4, Kappa CCD).

General procedure for the preparation of compounds $4 a, 4 c-n, 4 q, 4 b-1,4 e-1,4 f-1,4 g-1,40-1$ and $4 p-1$. $\mathrm{Sm}(\mathrm{OTf})_{3}(60 \mathrm{mg}, 0.1 \mathrm{mmol})$ was added to a solution of $3(1.0 \mathrm{mmol})$ in $\mathrm{MeNO}_{2}(5 \mathrm{~mL})$ at $25^{\circ} \mathrm{C}$. The reaction mixture was stirred at $25{ }^{\circ} \mathrm{C}$ for $10 \mathrm{~h}$. The solvent of reaction mixture was concentrated and extracted with $\mathrm{CH}_{2} \mathrm{Cl}_{2}(3 \times 10 \mathrm{~mL})$. The combined organic layers were washed with brine $(2 \times 10 \mathrm{~mL})$, dried $\left(\mathrm{MgSO}_{4}\right)$, filtered and evaporated to afford crude product mixture under reduced pressure. The remaining mixture was separated by flash column chromatography (silica gel, eluent: hexanes/EtOAc 100:1 $\rightarrow$ 20:1) affording compounds 4a, 4c-n, 4q, 4b-1, 4e-1, 4f-1, 4g-1, 4o-1 and 4p-1.

1,1,3-Trimethyl-3-phenylindan (4a). ${ }^{7}$ Colorless oil (98 mg, 83\%); HRMS (ESI-TOF) $\mathrm{m} / \mathrm{z}:[\mathrm{M}+\mathrm{H}]^{+}$calcd for $\mathrm{C}_{18} \mathrm{H}_{21}$ 237.1643, found 237.1644; ${ }^{1} \mathrm{H}$ NMR $\left(400 \mathrm{MHz}, \mathrm{CDCl}_{3}\right): \delta$ 7.45-7.25 (m, 9H), $2.58(\mathrm{~d}, J 13.2,1 \mathrm{H}), 2.35(\mathrm{~d}, J$ 13.2, 1H), $1.84(\mathrm{~s}, 3 \mathrm{H}), 1.50(\mathrm{~s}, 3 \mathrm{H}), 1.19(\mathrm{~s}, 3 \mathrm{H}) ;{ }^{13} \mathrm{C} \mathrm{NMR}\left(100 \mathrm{MHz}, \mathrm{CDCl}_{3}\right): \delta 152.1,151.0,148.7,128.0(2 \times)$, $127.2,126.64(2 \times), 126.61,125.5,125.0,122.5,59.2,50.8,42.8,30.9,30.7,30.4$.

4-Methyl-2,4-di(4-trifluoromethylphenyl)-1-pentene (4b-1). Colorless oil (108 mg, 58\%); HRMS (ESI-TOF) $\mathrm{m} / \mathrm{z}:[\mathrm{M}+\mathrm{H}]^{+}$calcd for $\mathrm{C}_{20} \mathrm{H}_{19} \mathrm{~F}_{6} 373.1391$, found 373.1396; ${ }^{1} \mathrm{H} \mathrm{NMR}\left(400 \mathrm{MHz}, \mathrm{CDCl}_{3}\right): \delta 7.38-7.34(\mathrm{~m}, 4 \mathrm{H})$, $7.27\left(\mathrm{~d}, J\right.$ 8.4, 2H), $7.16\left(\mathrm{~d}, J\right.$ 8.4, 2H), $5.18(\mathrm{~d}, J 1.2,1 \mathrm{H}), 4.95(\mathrm{~s}, 1 \mathrm{H}), 2.86(\mathrm{~s}, 2 \mathrm{H}), 1.30(\mathrm{~s}, 6 \mathrm{H}) ;{ }^{13} \mathrm{C} \mathrm{NMR}(100$ $\mathrm{MHz}, \mathrm{CDCl}_{3}$ ): $\delta$ 152.3, 146.3, 145.5, 128.9 (d, J 32.6), 127.9 (d, J 31.9), $126.8(2 \times), 126.4(2 \times), 124.2$ (q, J 269.8), 124.1 (d, J 269.9), 124.8 (q, J 3.8, 2x), 124.6 (d, J 3.8, 2x), 118.8, 50.0, 38.7, 28.6 (2x).

1,1,3,5-Tetramethyl-3-p-tolylindan (4c). ${ }^{16}$ Colorless oil (96 mg, 73\%); HRMS (ESI-TOF) $\mathrm{m} / \mathrm{z}$ : [M + H] ${ }^{+}$calcd for $\mathrm{C}_{20} \mathrm{H}_{25}$ 265.1956, found 265.1953; ${ }^{1} \mathrm{H}$ NMR $\left(400 \mathrm{MHz}, \mathrm{CDCl}_{3}\right): \delta$ 7.20-7.17 (m, 6H), $7.02(\mathrm{~s}, 1 \mathrm{H}), 2.49(\mathrm{~d}, J 13.2$, $1 \mathrm{H}), 2.46(\mathrm{~s}, 3 \mathrm{H}), 2.40(\mathrm{~s}, 3 \mathrm{H}), 2.28(\mathrm{~d}, J 13.2,1 \mathrm{H}), 1.78(\mathrm{~s}, 3 \mathrm{H}), 1.44(\mathrm{~s}, 3 \mathrm{H}), 1.16(\mathrm{~s}, 3 \mathrm{H}) ;{ }^{13} \mathrm{C} \mathrm{NMR}(100 \mathrm{MHz}$, $\left.\mathrm{CDCl}_{3}\right): \delta 149.3,149.1,148.1,136.1,134.8,128.6(2 \times), 128.0,126.6(2 \times), 125.4,122.2,59.5,50.3,42.5,30.8$ $(2 \times), 30.5,21.4,20.9$.

5-Methoxy-1-(3-methoxyphenyl)-1,3,3-trimethylindan (4d) and 4-methoxy-3-(3-methoxyphenyl)-1,1,3trimethylindan (4d'). ${ }^{18}$ Unseparated binary mixture; ratio 4d/4d' 80:20; Colorless oil (112 mg, 76\%); HRMS (ESI-TOF) $\mathrm{m} / \mathrm{z}$ : $[\mathrm{M}+\mathrm{H}]^{+}$calcd for $\mathrm{C}_{20} \mathrm{H}_{25} \mathrm{O}_{2}$ 297.1855, found 297.1856; for major product 4d, ${ }^{1} \mathrm{H} \mathrm{NMR}(400 \mathrm{MHz}$, $\left.\mathrm{CDCl}_{3}\right): \delta 7.20(\mathrm{t}, J 8.0,1 \mathrm{H}), 7.08(\mathrm{~d}, J 8.0,1 \mathrm{H}), 6.86-6.70(\mathrm{~m}, 5 \mathrm{H}), 3.87(\mathrm{~s}, 3 \mathrm{H}), 3.78(\mathrm{~s}, 3 \mathrm{H}), 2.46(\mathrm{~d}, J 12.8,1 \mathrm{H})$, $2.22(\mathrm{~d}, \mathrm{~J} 13.2,1 \mathrm{H}), 1.69(\mathrm{~s}, 3 \mathrm{H}), 1.36(\mathrm{~s}, 3 \mathrm{H}), 1.06$ (s, 3H); ${ }^{13} \mathrm{C} N M R\left(100 \mathrm{MHz}^{\mathrm{C}} \mathrm{CDCl}_{3}\right): \delta$ 159.4, 159.2, 153.7, 
153.1, 140.6, 128.8, 125.6, 119.3, 113.3, 112.5, 109.9, 107.7, 59.4, 55.3, 55.0, 50.1, 42.8, 31.1, 30.4, 30.2; GCMS: $m / z$ (\%) 297 (65) [M + H ], 282 (45), 267 (12), 236 (10), 158 (24), 144 (23), 91 (10).

5-Chloro-3-(4-chlorophenyl)-1,1,3-trimethylindan (4e). ${ }^{7}$ Colorless solid (102 mg, 67\%); mp 81-82 ${ }^{\circ} \mathrm{C}$ (from hexanes and EtOAc); HRMS (ESI-TOF) $\mathrm{m} / \mathrm{z}$ : $[\mathrm{M}+\mathrm{H}]^{+}$calcd for $\mathrm{C}_{18} \mathrm{H}_{19} \mathrm{Cl}_{2} 305.0864$, found 305.0866; ${ }^{1} \mathrm{H} \mathrm{NMR}(400$ $\left.\mathrm{MHz}, \mathrm{CDCl}_{3}\right): \delta$ 7.27-7.20 (m, 3H), 7.13-7.07 (m, 3H), $7.04(\mathrm{~d}, J 2.0,1 \mathrm{H}), 2.37(\mathrm{~d}, J 12.8,1 \mathrm{H}), 2.20(\mathrm{~d}, J 12.8,1 \mathrm{H})$, $1.65(\mathrm{~s}, 3 \mathrm{H}), 1.32(\mathrm{~s}, 3 \mathrm{H}), 1.03(\mathrm{~s}, 3 \mathrm{H}) ;{ }^{13} \mathrm{C} \mathrm{NMR}\left(100 \mathrm{MHz}, \mathrm{CDCl}_{3}\right): \delta 150.6,150.3,148.7,132.4,131.5,128.2$ (2x), $128.0(2 \times), 127.7,124.9,123.9,59.2,50.5,42.6,30.59,30.57,30.3$.

2,4-Di(4-chlorophenyl)-4-methyl-1-pentene (4e-1). ${ }^{42}$ Colorless oil (12 mg, 8\%); HRMS (ESI-TOF) $\mathrm{m} / \mathrm{z}:[\mathrm{M}+$ $\mathrm{H}^{+}$calcd for $\mathrm{C}_{18} \mathrm{H}_{19} \mathrm{Cl}_{2}$ 305.0864, found 305.0868; ${ }^{1} \mathrm{H}$ NMR $\left(400 \mathrm{MHz}, \mathrm{CDCl}_{3}\right): \delta$ 7.19-7.15 $(\mathrm{m}, 6 \mathrm{H}), 7.12-7.08(\mathrm{~m}$, $2 \mathrm{H}), 5.12(\mathrm{~d}, J 1.6,1 \mathrm{H}), 4.08(\mathrm{~d}, J 0.4,1 \mathrm{H}), 2.77(\mathrm{~s}, 2 \mathrm{H}), 1.22(\mathrm{~s}, 6 \mathrm{H}) ;{ }^{13} \mathrm{C} \mathrm{NMR}\left(100 \mathrm{MHz}, \mathrm{CDCl}_{3}\right): \delta 147.3,145.4$, 141.5, 132.7, 131.3, 128.1 (2x), $127.79(2 \times), 127.76(2 \times), 127.4(2 \times), 117.5,49.7,38.4,28.8(2 \times)$.

5-Fluoro-3-(4-fluorophenyl)-1,1,3-trimethylindan (4f). ${ }^{18}$ Colorless oil (90 mg, 66\%); HRMS (ESI-TOF) $\mathrm{m} / \mathrm{z}$ : [M $+\mathrm{H}^{+}$calcd for $\mathrm{C}_{18} \mathrm{H}_{19} \mathrm{~F}_{2} 273.1455$, found 273.1456; ${ }^{1} \mathrm{H} N M R\left(400 \mathrm{MHz}, \mathrm{CDCl}_{3}\right): \delta 7.15-7.11(\mathrm{~m}, 3 \mathrm{H}), 7.00-6.91$ $(\mathrm{m}, 3 \mathrm{H}), 6.76(\mathrm{dd}, J$ 2.4, 9.2, 1H), $2.39(\mathrm{~d}, J$ 12.8, 1H), $2.22(\mathrm{~d}, J 13.2,1 \mathrm{H}), 1.66(\mathrm{~s}, 3 \mathrm{H}), 1.33(\mathrm{~s}, 3 \mathrm{H}), 1.04(\mathrm{~s}, 3 \mathrm{H})$; ${ }^{13} \mathrm{C} \mathrm{NMR}\left(100 \mathrm{MHz}, \mathrm{CDCl}_{3}\right.$ ): $\delta 162.3$ (d, J 242.6), 161.0 (d, J 243.3), 150.7 (d, J 6.8), 147.5 (d, J 2.3), 146.1 (d, J 3.0), $128.0(d, J 7.6,2 \times), 123.7(d, J$ 8.3), $114.7(d, J$ 20.4, 2x), $114.4(d, J$ 22.7), $111.4(d, J$ 21.2), 59.6, 50.2, $42.4,30.8,30.7,30.4$.

2,4-Di(4-fluorophenyl)-4-methyl-1-pentene (4f-1). ${ }^{42}$ Colorless oil (20 mg, 15\%); HRMS (ESI-TOF) $\mathrm{m} / \mathrm{z}$ : [M + $\mathrm{H}]^{+}$calcd for $\mathrm{C}_{18} \mathrm{H}_{19} \mathrm{~F}_{2} 273.1455$, found 273.1458; ${ }^{1} \mathrm{H}$ NMR $\left(400 \mathrm{MHz}, \mathrm{CDCl}_{3}\right): \delta$ 6.85-6.76 (m, 4H), 6.56-6.49 (m, $4 \mathrm{H}), 4.74(\mathrm{~d}, J 1.6,1 \mathrm{H}), 4.44(\mathrm{~d}, J 0.8,1 \mathrm{H}), 2.43(\mathrm{~s}, 2 \mathrm{H}), 0.88(\mathrm{~s}, 6 \mathrm{H}) ;{ }^{13} \mathrm{CNMR}\left(100 \mathrm{MHz}, \mathrm{CDCl}_{3}\right): \delta 161.9(\mathrm{~d}, J$ 244.1), 160.8 (d, J 242.6), 145.6, 144.5, 139.1, 128.0 (d, J 7.6, 2x), 127.4 (d, J 7.6, 2x), 116.9, 114.7 (d, J 21.2, $2 \times), 114.3(d, J 20.5,2 x), 50.1,38.5,29.0(2 x)$.

5-Fluoro-1-(3-fluorophenyl)-1,3,3-trimethylindan (4g). Colorless oil (83 mg, 61\%); HRMS (ESI-TOF) $\mathrm{m} / \mathrm{z}$ : [M + $\mathrm{H}]^{+}$calcd for $\mathrm{C}_{18} \mathrm{H}_{19} \mathrm{~F}_{2} 273.1455$, found 273.1457; ${ }^{1} \mathrm{H}$ NMR $\left(400 \mathrm{MHz}, \mathrm{CDCl}_{3}\right): \delta$ 7.23-7.17 (m, $\left.1 \mathrm{H}\right), 7.05(\mathrm{~d}, J 8.4$, $1 \mathrm{H}), 7.04(\mathrm{~d}, J$ 8.4, $1 \mathrm{H}), 6.97-6.91(\mathrm{~m}, 2 \mathrm{H}), 6.88-6.82(\mathrm{~m}, 2 \mathrm{H}), 2.41(\mathrm{~d}, J 13.2,1 \mathrm{H}), 2.22(\mathrm{~d}, J 13.2,1 \mathrm{H}), 1.65(\mathrm{~s}$, 3H), 1.33 (s, 3H), 1.02 (s, 3H); $\left.{ }^{13} \mathrm{C} \mathrm{NMR} \mathrm{(100} \mathrm{MHz,} \mathrm{CDCl}\right): \delta 164.0$ (d, J 242.6), 162.7 (d, J 243.3), 154.5 (d, J 6.8 ), 153.6 (d, J 5.3), 143.4 (d, J 3.0), 129.4 (d, J 8.3), 125.9 (d, J 8.4), 122.2 (d, J 3.0), 113.9 (d, J 19.7), 113.7 (d, J 19.0), 112.4 (d, J 20.5), 109.5 (d, J 21.2), 59.3, 50.3, 42.9, 30.9, 30.4, 30.1.

2,4-Di(3-fluorophenyl)-4-methyl-1-pentene (4g-1). Colorless oil (24 mg, 18\%); HRMS (ESI-TOF) $\mathrm{m} / \mathrm{z}$ : [M + H] calcd for $\mathrm{C}_{18} \mathrm{H}_{19} \mathrm{~F}_{2} 273.1455$, found 273.1455; ${ }^{1} \mathrm{H}$ NMR $\left(400 \mathrm{MHz}, \mathrm{CDCl}_{3}\right): \delta 7.18-7.12(\mathrm{~m}, 2 \mathrm{H}), 7.03-7.01(\mathrm{~m}, 1 \mathrm{H})$, 6.98-6.96 (m, 1H), 6.94-6.90 (m, 1H), 6.88-6.83 (m, 2H), 6.80-6.75 (m, 1H), 5.16 (d, J 1.6, 1H), $4.84(\mathrm{~s}, 1 \mathrm{H}), 2.78$ (s, 2H), $1.24(\mathrm{~s}, 6 \mathrm{H}) ;{ }^{13} \mathrm{C} \mathrm{NMR}\left(100 \mathrm{MHz}, \mathrm{CDCl}_{3}\right): \delta 162.6(\mathrm{~d}, J 242.6), 162.5(\mathrm{~d}, J 242.6), 151.7(\mathrm{~d}, J 8.8), 145.4(\mathrm{~d}$, J 2.3), 138.9 (d, J 8.2), 129.4 (d, J 8.3), 129.1 (d, J 8.3), 122.1 (d, J 2.3), 121.6 (d, J 2.3), 117.9, 113.6 (d, J 20.5), 113.3 (d, J 22.0), 113.2 (d, J 21.2), 112.3 (d, J 21.2), 49.6, 38.7, $28.6(2 \times)$.

1-(3,4-Dimethoxyphenyl)-5,6-dimethoxy-1,3,3-trimethylindan (4h). ${ }^{19}$ Colorless oil (134 mg, 75\%); HRMS (ESI-TOF) $\mathrm{m} / \mathrm{z}$ : $[\mathrm{M}+\mathrm{H}]^{+}$calcd for $\mathrm{C}_{22} \mathrm{H}_{29} \mathrm{O}_{4} 357.2066$, found $357.2067 ;{ }^{1} \mathrm{H} \mathrm{NMR}\left(400 \mathrm{MHz}, \mathrm{CDCl}_{3}\right): \delta 6.74-6.69$ $(\mathrm{m} \mathrm{4H}), 6.60(\mathrm{~s}, 1 \mathrm{H}), 3.92(\mathrm{~s}, 3 \mathrm{H}), 3.84(\mathrm{~s}, 3 \mathrm{H}), 3.83(\mathrm{~s}, 3 \mathrm{H}), 3.78(\mathrm{~s}, 3 \mathrm{H}), 2.34(\mathrm{~d}, J 13.2,1 \mathrm{H}), 2.17(\mathrm{~d}, J 13.2,1 \mathrm{H})$, $1.66(\mathrm{~s}, 3 \mathrm{H}), 1.32(\mathrm{~s}, 3 \mathrm{H}), 1.05(\mathrm{~s}, 3 \mathrm{H}) ;{ }^{13} \mathrm{C} \mathrm{NMR}\left(100 \mathrm{MHz}, \mathrm{CDCl}_{3}\right): \delta 148.7,148.3,148.2,146.7,143.9,143.8$, $140.1,118.5,110.38,110.36,107.4,105.1,59.7,56.0,55.9,55.7(2 \times), 50.3,42.7,30.8,30.7,30.4$.

5-Benzo[1,3]dioxol-5-yl-5,7,7-trimethyl-6,7-dihydro-5H-indeno[5,6-d][1,3]dioxole (4i). ${ }^{20}$ Colorless oil (126 $\mathrm{mg}, 78 \%$ ); HRMS (ESI-TOF) $\mathrm{m} / \mathrm{z}$ : $[\mathrm{M}+\mathrm{H}]^{+}$calcd for $\mathrm{C}_{20} \mathrm{H}_{21} \mathrm{O}_{4} 325.1440$, found 325.1438; ${ }^{1} \mathrm{H} \mathrm{NMR}(400 \mathrm{MHz}$, $\left.\mathrm{CDCl}_{3}\right): \delta 6.71-6.62(\mathrm{~m}, 4 \mathrm{H}), 6.53(\mathrm{~s}, 1 \mathrm{H}), 5.96(\mathrm{~d}, J$ 1.6, $1 \mathrm{H}), 5.95(\mathrm{~d}, J 1.2,1 \mathrm{H}), 5.91(\mathrm{~d}, J$ 2.0, $1 \mathrm{H}), 5.91(\mathrm{~d}, J 1.6$, $1 \mathrm{H}), 2.34(\mathrm{~d}, J 13.2,1 \mathrm{H}), 2.16(\mathrm{~d}, J \mathrm{~J} 2.8,1 \mathrm{H}), 1.61(\mathrm{~s}, 3 \mathrm{H}), 1.29(\mathrm{~s}, 3 \mathrm{H}), 1.05(\mathrm{~s}, 3 \mathrm{H}) ;{ }^{13} \mathrm{C} \mathrm{NMR}\left(100 \mathrm{MHz}^{\mathrm{CDCl}}\right)_{3}: \delta$ 
$147.4,147.2,146.7,145.3,145.2(2 \times), 141.5,119.4,107.6,107.4,104.9,102.8,101.0,100.8,59.7,50.3,42.6$, $30.93,30.87,30.4$.

4,5,6-Trimethoxy-1,1,3-trimethyl-3-(3,4,5-trimethoxyphenyl)indan (4j). ${ }^{20}$ Colorless oil (166 mg, 80\%); HRMS (ESI-TOF) $\mathrm{m} / \mathrm{z}:[\mathrm{M}+\mathrm{H}]^{+}$calcd for $\mathrm{C}_{24} \mathrm{H}_{33} \mathrm{O}_{6} 417.2277$, found 417.2273; ${ }^{1} \mathrm{H} \mathrm{NMR}\left(400 \mathrm{MHz}, \mathrm{CDCl}_{3}\right): \delta 6.46$ $(\mathrm{s}, 1 \mathrm{H}), 6.40(\mathrm{~s}, 2 \mathrm{H}), 3.89(\mathrm{~s}, 3 \mathrm{H}), 3.82(\mathrm{~s}, 3 \mathrm{H}), 3.80(\mathrm{~s}, 3 \mathrm{H}), 3.75(\mathrm{~s}, 6 \mathrm{H}), 3.60(\mathrm{~s}, 3 \mathrm{H}), 2.32(\mathrm{~d}, J 13.2,1 \mathrm{H}), 2.12(\mathrm{~d}$, J 12.8, 1H), $1.76(\mathrm{~s}, 3 \mathrm{H}), 1.30(\mathrm{~s}, 3 \mathrm{H}), 1.07(\mathrm{~s}, 3 \mathrm{H}) ;{ }^{13} \mathrm{C} \mathrm{NMR}\left(100 \mathrm{MHz}, \mathrm{CDCl}_{3}\right): \delta$ 153.8, $152.4(2 \times), 150.2,147.7$, 146.9, 141.0, 135.7, 132.8, 103.9 (2x), 100.8, 60.8, 60.5, 60.1, 60.0, 56.0, 55.9 (2x), 51.0, 43.4, 31.0, 30.5, 29.1.

4,5',6,7'-Tetramethoxy-3,3,3',3'-tetramethyl-2,2',3,3'-tetrahydro-1,1'-spirobi[indene] (4k). Colorless solid (150 mg, 76\%); mp 154-155 ${ }^{\circ} \mathrm{C}$ (from hexanes and EtOAc); HRMS (ESI-TOF) $\mathrm{m} / \mathrm{z}:[\mathrm{M}+\mathrm{H}]^{+}$calcd for $\mathrm{C}_{25} \mathrm{H}_{33} \mathrm{O}_{4}$ 397.2379, found 397.2381; ${ }^{1} \mathrm{H}$ NMR $\left(400 \mathrm{MHz}, \mathrm{CDCl}_{3}\right): \delta 6.33(\mathrm{~d}, J 2.0,1 \mathrm{H}), 6.27$ (d, J 2.0, $\left.1 \mathrm{H}\right), 6.26(\mathrm{~d}, J 2.0$, $1 \mathrm{H}), 5.91(\mathrm{~d}, J 2.0,1 \mathrm{H}), 3.84(\mathrm{~s}, 3 \mathrm{H}), 3.81(\mathrm{~s}, 3 \mathrm{H}), 3.67(\mathrm{~s}, 3 \mathrm{H}), 3.51(\mathrm{~s}, 3 \mathrm{H}), 2.53(\mathrm{~d}, J 12.8,1 \mathrm{H}), 2.28(\mathrm{~d}, J 13.2$, $1 \mathrm{H}), 2.14(\mathrm{~d}, J 13.2,1 \mathrm{H}), 2.07(\mathrm{~d}, J 12.8,1 \mathrm{H}), 1.45(\mathrm{~s}, 3 \mathrm{H}), 1.40(\mathrm{~s}, 3 \mathrm{H}), 1.36(\mathrm{~s}, 3 \mathrm{H}), 1.30(\mathrm{~s}, 3 \mathrm{H}) ;{ }^{13} \mathrm{C} \mathrm{NMR}(100$ $\left.\mathrm{MHz}_{1} \mathrm{CDCl}_{3}\right): \delta 160.9,160.2,156.9,156.6,155.2,154.1,130.0,128.0,98.9,97.9,97.6,96.8,60.5,57.1,56.7$, $55.43,55.37,55.1,54.9,44.1,43.5,31.9,30.0,29.0,28.9$. Single-crystal X-Ray diagram: crystal of compound $\mathbf{4 k}$ was grown by slow diffusion of EtOAc into a solution of compound $\mathbf{4} \mathbf{k}$ in $\mathrm{CH}_{2} \mathrm{Cl}_{2}$ to yield colorless prisms. The compound crystallizes in the triclinic crystal system, space group P $-1, a=9.4024(3) \AA, b=15.5199(5) \AA, c=$ 16.4035(6) $\AA, V=2163.91(13) \AA^{3}, Z=2, d_{\text {calcd }}=1.217 \mathrm{~g} / \mathrm{cm}^{3}, F(000)=856,2 \vartheta$ range $1.328^{\sim} 26.411^{\circ}$, R indices (all data) $\mathrm{R} 1=0.0976, \mathrm{wR} 2=0.2137$.

4,6,6-Trimethyl-4-thien-2-yl-5,6-dihydro-4H-cyclopenta[b]thiophene (4l). Colorless oil (81 mg, 65\%); HRMS (ESI-TOF) $\mathrm{m} / \mathrm{z}:[\mathrm{M}+\mathrm{H}]^{+}$calcd for $\mathrm{C}_{14} \mathrm{H}_{17} \mathrm{~S}_{2}$ 249.0772, found 249.0773; ${ }^{1} \mathrm{H} \mathrm{NMR}\left(400 \mathrm{MHz}, \mathrm{CDCl}_{3}\right): \delta 7.21$ (d, $J$ 4.8, 1H), $7.12(\mathrm{dd}, J$ 0.8, 4.8, 1H), $6.87(\mathrm{dd}, J 3.6,4.8,1 \mathrm{H}), 6.78(\mathrm{~d}, J 4.8,1 \mathrm{H}), 6.69(\mathrm{dd}, J$ J.8, 3.6, $1 \mathrm{H}), 2.72(\mathrm{~d}, J$ $12.8,1 \mathrm{H}), 2.54(\mathrm{~d}, \mathrm{~J} 13.2,1 \mathrm{H}), 1.72(\mathrm{~s}, 3 \mathrm{H}), 1.40(\mathrm{~s}, 3 \mathrm{H}), 1.28(\mathrm{~s}, 3 \mathrm{H}) ;{ }^{13} \mathrm{C} \mathrm{NMR}\left(100 \mathrm{MHz}, \mathrm{CDCl}_{3}\right): \delta 152.3,149.6$, $127.9,126.4,123.0,122.5,121.3,121.2,64.0,46.8,42.3,31.8,31.6,31.3$.

4-(1,3,3-Trimethyl-6-phenylindan-1-yl)biphenyl (4m). ${ }^{8}$ Colorless solid (134 mg, 69\%); mp $102-103{ }^{\circ} \mathrm{C}$ (from hexanes and EtOAc); HRMS (ESI-TOF) $\mathrm{m} / \mathrm{z}$ : $[\mathrm{M}+\mathrm{H}]^{+}$calcd for $\mathrm{C}_{30} \mathrm{H}_{29} 389.2269$, found 389.2271; ${ }^{1} \mathrm{H} \mathrm{NMR}(400$ $\mathrm{MHz}, \mathrm{CDCl}_{3}$ ): $\delta$ 7.66-7.32 (m, 17H), $2.54(\mathrm{~d}, J 13.2,1 \mathrm{H}), 2.32$ (d, J 12.8, 1H), $1.82(\mathrm{~s}, 3 \mathrm{H}), 1.44(\mathrm{~s}, 3 \mathrm{H}), 1.16(\mathrm{~s}, 3 \mathrm{H})$ ${ }^{13} \mathrm{C} \mathrm{NMR}\left(100 \mathrm{MHz}, \mathrm{CDCl}_{3}\right): \delta 151.5,150.0,149.4,141.6,140.9,140.0,138.3,128.7(4 \mathrm{x}), 127.2(2 \times), 127.1(2 \times)$, $127.0(2 \times), 126.9(2 \times), 126.7(2 \times), 126.5,123.7,122.9,59.4,50.7,42.7,30.9,30.7,30.5$.

1,3,3-Trimethyl-1-naphthalen-2-yl-2,3-dihydro-1H-cyclopenta[a]naphthalene $(\mathbf{4 n}) .^{21}$ Colorless solid (118 $\mathrm{mg}, 70 \%$ ); $\mathrm{mp} 117-118^{\circ} \mathrm{C}$ (from hexanes and EtOAc); HRMS (ESI-TOF) $\mathrm{m} / \mathrm{z}$ : [M + H] ${ }^{+}$calcd for $\mathrm{C}_{26} \mathrm{H}_{25} 337.1956$, found 337.1952; ${ }^{1} \mathrm{H} \mathrm{NMR}\left(400 \mathrm{MHz}, \mathrm{CDCl}_{3}\right): \delta$ 7.97-7.84 (m, 5H), $7.74(\mathrm{~d}, J$ 8.8, $1 \mathrm{H}), 7.56-7.49(\mathrm{~m}, 4 \mathrm{H}), 7.38(\mathrm{dt}$, J 1.2, 8.0, 1H), 7.33 (dd, J 2.0, 8.8, 1H), $7.19(\mathrm{dt}, J 1.2,8.4,1 \mathrm{H}), 2.58(\mathrm{~d}, J 13.6,1 \mathrm{H}), 2.46(\mathrm{~d}, J 13.2,1 \mathrm{H}), 2.19(\mathrm{~s}$, $3 \mathrm{H}), 1.56(\mathrm{~s}, 3 \mathrm{H}), 1.51(\mathrm{~s}, 3 \mathrm{H}) ;{ }^{13} \mathrm{C}$ NMR $\left(100 \mathrm{MHz} \mathrm{CDCl}_{3}\right): \delta 149.4,148.6,142.7,133.9,133.3,131.7,129.8$, $128.8,128.7,128.1,128.0,127.4,126.3,125.8,125.6,125.3,125.0,124.5,123.5,121.3,61.4,52.1,43.3,31.4$, $31.3,28.1$.

4-Methyl-2,4-di(4-nitrophenyl)-1-pentene (4o-1). Colorless solid (104 mg, 64\%); mp $124-125{ }^{\circ} \mathrm{C}$ (from hexanes and EtOAc); HRMS (ESI-TOF) $\mathrm{m} / \mathrm{z}$ : $[\mathrm{M}+\mathrm{H}]^{+}$calcd for $\mathrm{C}_{18} \mathrm{H}_{19} \mathrm{~N}_{2} \mathrm{O}_{4} 327.1345$ found 327.1345; ${ }^{1} \mathrm{H} N M R$ $\left(400 \mathrm{MHz}, \mathrm{CDCl}_{3}\right): \delta 8.08-8.02(\mathrm{~m}, 4 \mathrm{H}), 7.39(\mathrm{~d}, J$ 8.8, 2H), $7.31(\mathrm{~d}, J$ 8.8, 2H), $5.26(\mathrm{~d}, J 1.2,1 \mathrm{H}), 4.96(\mathrm{~d}, J 0.8$, $1 \mathrm{H}), 2.90(\mathrm{~s}, 2 \mathrm{H}), 1.29(\mathrm{~s}, 6 \mathrm{H}) ;{ }^{13} \mathrm{C} \mathrm{NMR}\left(100 \mathrm{MHz}, \mathrm{CDCl}_{3}\right): \delta 156.0,149.4,146.8,146.0,144.3,127.2(2 \times), 127.0$ $(2 \times), 123.4(2 x), 123.1(2 x), 120.7,49.4,39.4,28.7(2 x)$.

4-Methyl-2,4-di(3,4-dichlorophenyl)-1-pentene (4p-1). ${ }^{43}$ Colorless oil (121 mg, 65\%); HRMS (ESI-TOF) $\mathrm{m} / \mathrm{z}$ : $[\mathrm{M}+\mathrm{H}]^{+}$calcd for $\mathrm{C}_{18} \mathrm{H}_{17} \mathrm{Cl}_{4} 373.0084$, found 373.0086; ${ }^{1} \mathrm{H} \mathrm{NMR}\left(400 \mathrm{MHz}, \mathrm{CDCl}_{3}\right): \delta 7.23(\mathrm{~d}, J 8.4,1 \mathrm{H}), 7.21(\mathrm{~d}$, J 8.4, 1H), $7.18(\mathrm{~d}, J 2.4,1 \mathrm{H}), 7.09(\mathrm{~d}, J$ 2.4, 1H), 7.01 (dd, J 2.6, 8.4, 1H), $6.92(\mathrm{dd}, J 2.0,8.4,1 \mathrm{H}), 5.15(\mathrm{~d}, J 1.2$, 
1H), 4.91 (d, J 1.2, 1H), $2.72(\mathrm{~s}, 2 \mathrm{H}), 1.25(\mathrm{~s}, 6 \mathrm{H}) ;{ }^{13} \mathrm{C} \mathrm{NMR}\left(100 \mathrm{MHz}, \mathrm{CDCl}_{3}\right): \delta$ 148.5, 144.3, 142.6, 132.0, $131.8,130.8,129.7,129.6,129.5,128.5,128.3,125.8,125.6,118.5,49.9,38.3,28.5$ (2x).

1-Methyl-1,3,3-triphenylindan (4q). ${ }^{18}$ Colorless oil (101 mg, 56\%); HRMS (ESI-TOF) $\mathrm{m} / \mathrm{z}:[\mathrm{M}+\mathrm{H}]^{+}$calcd for $\mathrm{C}_{28} \mathrm{H}_{25}$ 361.1956, found 361.1958; ${ }^{1} \mathrm{H}$ NMR (400 MHz, CDCl $): \delta 7.31-7.01(\mathrm{~m}, 19 \mathrm{H}), 3.40(\mathrm{~d}, J 13.6,1 \mathrm{H}), 3.10(\mathrm{~d}$, J 13.2, 1H), $1.55(\mathrm{~s}, 3 \mathrm{H}) ;{ }^{13} \mathrm{CNMR}\left(100 \mathrm{MHz}, \mathrm{CDCl}_{3}\right): \delta 150.5,149.3,148.8,148.5,147.5,128.8(2 \times), 128.7(2 \times)$, $127.9(2 \times), 127.8(2 \times), 127.6(2 \times), 127.4,127.3,126.9(2 \times), 126.8,126.0,125.64,125.57,125.0,61.3,60.9$, $51.2,31.9$.

\section{Acknowledgements}

The authors thank the Ministry of Science and Technology of the Republic of China for financial support (MOST 106-2628-M-037-001-MY3).

\section{Supplementary Material}

Scanned photocopies of $\mathrm{NMR}\left(\mathrm{CDCl}_{3}\right)$ spectral data were supported.

\section{References}

1. Bergmann, E.; Taubadel, H.; Weib, H. Chem. Ber. 1931, 64, 1493-1501. https://doi.org/10.1002/cber.19310640641

2. Stavber, G.; Zupan, M.; Stavber, S. Tetrahedron Lett. 2006, 47, 8463-8466. https://doi.org/10.1016/i.tetlet.2006.09.154

3. Hojo, M.; Tadaaki, I.; Kishio, S. J. Org. Chem. 1985, 50, 1478-1482. https://doi.org/10.1021/jo00209a024

4. Ma, H.; Sun, Q.; Li, W.; Wang, J.; Zhang, Z.; Yang, Y.; Lei, Z. Tetrahedron Lett. 2011, 52, 1569-1573. https://doi.org/10.1016/i.tetlet.2011.01.056

5. Ciminale, F.; Lopez, L.; Paradiso, V.; Nacci, A. Tetrahedron 1996, 52, 13971-13980. https://doi.org/10.1016/0040-4020(96)00838-1

6. Peppe, C.; Lang, E. S.; de Andrade, F. M.; de Castro, L. B. Synlett 2004, 1723-1726. https://10.1055/s-2004-829557

7. Sun, H.-B.; Li, B.; Hua, R.; Yin, Y. Eur. J. Org. Chem. 2006, 4231-4236. https://doi/10.1002/ejoc.200600390

8. Miyake, Y.; Moriyama, T.; Tanabe, Y.; Onodera, G.; Nishibayashi, Y. Organometallics 2011, 30, 5972-5977. https://doi.org/10.1021/om200787s

9. Tsuchimoto, T.; Kamiyama, S.; Negoro, R.; Shirakawa, E.; Kawakami, Y. Chem. Commun. 2003, 852-853. https://10.1039/B301280H

10. Tomas, R. R.; Chebolu, V.; Sen, A. J. Am. Chem. Soc. 1986, 108, 4096-4103. https://doi.org/10.1021/ja00274a039

11. Hu, X.; Martin, D.; Melaimi, M.; Bertrand, G. J. Am. Chem. Soc. 2014, 136, 13594-13597. https://doi.org/10.1021/ja507788r 
12. Sen, A.; Lai, T.-W.; Thomas, R. R. J. Organomet. Chem. 1988, 358, 567-588. https://doi.org/10.1016/0022-328X(88)87104-3

13. Tateiwa, J.-i.; Kimura, A.; Takasuka, M.; Uemura, S. Bull. Chem. Soc. Jpn. 1996, 69, 2361-2368. https://doi.org/10.1246/bcsj.69.2361

14. Fujiwara, M.; Kuraoka, K.; Yazawa, T.; Xu, Q.; Tanaka, M.; Souma, Y. Chem. Commun. 2000, 1523-1524. https://10.1039/B004842I

15. Fujiwara, M.; Terashima, S.; Endo, Y.; Shiokawa, K.; Ohue, H. Chem. Commun. 2006, 4635-4637. https://10.1039/B610444D

16. Alwsso, E.; Torviso, R.; Erlich, M.; Finkielsztein, L.; Lantano, B.; Moltrasio, G.; Aquirre, J.; Vazquez, P.; Pizzio, L.; Caceres, C.; Blanco, M.; Thomas, H. Synth. Commun. 2002, 32, 3803-3812. https://doi.org/10.1081/SCC-120015399

17. Aun, Q.; Farneth, W. E.; Harmer, M. A. J. Catal. 1996, 164, 62-69. https://doi.org/10.1006/jcat.1996.0363

18. Alavala, R. G. K.; Satyanarayana, G. J. Org. Chem. 2016, 81, 12212-12222. https://doi.org/10.1021/acs.joc.6b02015

19. Reddel, J. C. T.; Wang, W.; Koukounas, K.; Thomson, R. J. Chem. Sci. 2017, 8, 2156-2160. https://10.1039/C6SC04762A

20. Hsueh, N.-C.; Chan, C.-K.; Chang, M.-Y. Tetrahedron 2018, 74, 1002-1008. https://doi.org/10.1016/i.tet.2018.01.023

21. Matsumoto, S.; Naito, M.; Oseki, T.; Akazome, M.; Otani, Y. Tetrahedron 2017, 73, 7254-7259. https://doi.org/10.1016/i.tet.2017.11.009

22. Chang, M.-Y.; Chen, Y.-C.; Chan, C.-K.; Huang, G. G. Tetrahedron 2015, 71, 2095-2104. https://doi.org/10.1016/j.tet.2015.02.046

23. Chang, M.-Y.; Chen, Y.-H.; Cheng, Y.-C. Tetrahedron 2016, 72, 518-524. https://doi.org/10.1016/i.tet.2015.12.007

24. Chang, M.-Y.; Cheng, Y.-C.; Lu, Y.-J. Org. Lett. 2015, 17, 1264-1267. https://doi.org/10.1021/acs.orglett.5b00246

25. Chang, M.-Y.; Cheng, Y.-C.; Lu, Y.-J. Org. Lett. 2015, 17, 3142-3145. https://doi.org/10.1021/acs.orglett.5b01461

26. Chang, M.-Y.; Cheng, Y.-C. Org. Lett. 2015, 17, 5702-5705. https://doi.org/10.1021/acs.orglett.5b03020

27. Chang, M.-Y.; Lu, Y.-J.; Cheng, Y.-C. Tetrahedron 2015, 71, 6840-6845. https://doi.org/10.1016/j.tet.2015.07.025

28. Chang, M.-Y.; Cheng, Y.-C. Synlett 2016, 27, 1931-1935. https://10.1055/s-0035-1561652

29. Ladziata, U. Arkivoc 2014, i, 307-336. http://dx.doi.org/10.3998/ark.5550190.p008.620

30. Kobayashi, S.; Sugiura, M.; Kitagawa, H.; Lam, W. W.-L. Chem. Rev. 2002, 102, 2227-2302. https://doi.org/10.1021/cr010289i

31. Chen, P.; Chang, D. Tetrahedron 2014, 70, 8505-8510. https://doi.org/10.1016/i.tet.2014.09.060

32. Tsuchimoto, T.; Tobita, K.; Hiyama, T.; Fukuzawa, S.-i. J. Org. Chem. 1997, 62, 6997-7005. https://doi.org/10.1021/jo970599u

33. Shang, D.; Xin, J.; Liu, Y.; Zhou, X.; Liu, X.; Feng, X. J. Org. Chem. 2008, 73, 630-637. 
https://doi.org/10.1021/jo7021263

34. Abe, A. M. M.; Sauerland, S. J. K.; Koskinen, A. M. P. J. Org. Chem. 2007, 72, 5411-5413. https://doi.org/10.1021/jo070492z

35. Wang, X.; Li, S.-y.; Pan, Y.-m.; Wang, H.-s.; Liang, H.; Chen, Z.-f.; Qin, X.-h. Org. Lett. 2014, 16, 580-583. https://doi.org/10.1021/ol4034513

36. Gopinath, P.; Nilaya, S.; Muraleedharan, K. M. Org. Lett. 2011, 13, 1932-1935. https://doi.org/10.1021/ol200247c

37. Saadi, J.; Lentz, D.; Reissig, H.-U. Org. Lett. 2009, 11, 3334-3337. https://doi.org/10.1021/ol901183h

38. Ueda, T.; Kanomata, N.; Machida, H. Org. Lett. 2005, 7, 2365-2368. https://doi.org/10.1021/ol0506258

39. James, K. M.; Willetts, N.; Procter, D. J. Org. Lett. 2008, 10, 1203-1206. https://doi.org/10.1021/ol800070y

40. Aspinall, H. C.; Greeves, N.; Valla, C. Org. Lett. 2005, 7, 1919-1922. https://doi.org/10.1021/ol050256f

41. CCDC 1864310 (4k) contains the supplementary crystallographic data for this paper. This data can be obtained free of charge via www.ccdc.cam.ac.uk/conts/retrieving.html (or from the CCDC, 12 Union Road, Cambridge CB2 1EZ, UK; fax: 44-1223-336033; e-mail: deposit@ccdc.cam.ac.uk).

42. Sanz, R.; Martinez, A.; Guilarte, V.; Alvarez-Gutierrez, J. M.; Rodriguez, F. Eur. J. Org. Chem. 2007, 46424645.

https://doi.org/10.1002/ejoc.200700562

43. Okachi, T.; Fujimoto, K.; Onaka, M. Org. Lett. 2002, 4, 1667-1669.

https://doi.org/10.1021/ol025719| 